\title{
Tolerance Redistributing of the Reassembly Dimensional Chain on Measure of Uncertainty
}

\author{
Conghu Liu ${ }^{1,2}$ \\ 1 School of Mechanical and Electrical Engineering, Suzhou University, Suzhou 234000, China; \\ lch339@126.com; Tel.: +86-557-2871-036 \\ 2 School of Mechanical and Automotive Engineering, Hefei University of Technology, Hefei 230009, China \\ Academic Editor: Adom Giffin \\ Received: 7 July 2016; Accepted: 19 September 2016; Published: 9 October 2016
}

\begin{abstract}
How to use the limited precision of remanufactured parts to assemble higher-quality remanufactured products is a challenge for remanufacturing engineering under uncertainty. On the basis of analyzing the uncertainty of remanufacturing parts, this paper takes tolerance redistributing of the reassembly (remanufactured assembly) dimensional chain as the research object. An entropy model to measure the uncertainty of assembly dimensional is built, and we quantify the degree of the uncertainty gap between reassembly and assembly. Then, in order to make sure the uncertainty of reassembly is not lower than that of assembly, the tolerance redistribution optimization model of the reassembly dimensional chain is proposed which is based on the tolerance of the grading allocation method. Finally, this paper takes the remanufactured gearbox assembly dimension chain as an example. The redistribution optimization model saves $19.11 \%$ of the cost with the assembly precision of remanufactured products. It provides new technical and theoretical support to expand the utilization rate of remanufactured parts and improve reassembly precision.
\end{abstract}

Keywords: remanufacturing; entropy; assembly dimensional; redistribution

\section{Introduction}

Faced with an increasingly prominent enormous pressure on natural resources and the ecological environment, remanufacturing has become one of the most important technologies for the world's sustainable economic development. Remanufacturing is the process of restoring the old waste and used products to a new state, and remanufactured products are expected to be of the same or better quality as the original products [1]. However, the remanufactured objects are recycled parts, which have a series of defects, such as poor dimensional, residual stress, internal cracks, surface deformation and so on. At the same time, the uncertainties of recycled parts require distinct remanufacturing repair processes and technologies. Compared with the manufactured products, the assembly dimensional of remanufactured parts have a higher uncertainty, which is shown in the change in the mean, the expansion of the variance, and the increase in the tolerance range. These uncertainties are produced, delivered and coupled during the reassembly process, and then affect the quality of the remanufactured products [2]. Therefore, how to measure the degree of uncertainty for remanufactured parts assembly dimensional with a unified dimensionless model has become one of the key issues in the process of remanufacturing assembly production management.

The existing research on the uncertainty of remanufactured parts shows that, due to the influence of different degrees of the thermal field and stress field in the remanufacturing repair process, the shape, hardness, strength and toughness of the remanufactured parts has appeared in the gradient of the tissue or the gradient of the performance [3,4]. Liu [5] considered that there is a greater degree of variance of the components, and there is a higher uncertainty with respect to the new parts, and the mathematical model of uncertainty measurement is established. Based on the analysis of the 
uncertainty of the surface roughness of the crankshaft of the engine, the measurement model of the roughness of the crankshaft journal surface was built by Liu [6]. Liu's research shows that this kind of remanufactured part and new products' manufactured parts are uncertain, but the uncertainty of remanufacturing parts is higher than that of the new products [7].

The study of assembly control in remanufacturing is as follows: Ge [8] studied the online quality control methods in the reassembly process for remanufactured engines' cylinder blocks and covers. The optimal control strategy of product reorganization in the remanufacturing system with variable quality returns was studied by Jin [9]. Liu [10,11] studied the matching method of different-quality-grade remanufactured parts, and proposed an assembly grouping optimal allocation method for remanufactured products.

The above research shows that the measurement of the uncertainty of remanufacturing assembly is a key index to quantify the degree of internal chaos in the remanufacturing assembly system, and also to evaluate the quality control system. Therefore, the uncertainty of remanufacturing parts is measured and used in the assembly system as a key to improve the quality of remanufacturing assembly. Information entropy [12] is a tool which is a measure of uncertainty and an applied research in the production system [13]. For example: Frizelle and Suhov [14] considered input-output processes as models of Kolmogorov-Sinai entropy for manufacturing systems. Efstathiou [15] proved that the system entropy is equal to the complexity of the manufacturing systems, and used the information-theoretic entropy to measure the structural, dynamic and decision-making complexity of a manufacturing system. Kuzgunkaya and ElMaraghy [16] used an entropy approach for assessing the structural complexity of manufacturing system configurations. Cho [17] proposed an analytical model for measuring the system complexity and he employed information entropy which can quantitatively assess the complexity of manufacturing systems in various configurations, including assembly and disassembly systems. Zhang [18] proposed a means of measuring the information demands and placed it on cellular manufacturing systems as a result of this uncertainty. Duan [19] established a complex model of non-serial manufacturing systems, which is based on the entropy model of information theory from the angle of the system and so on.

Considering the assembly dimension chain and remanufacturing machining capability, we found that technical means can be used to redistribute the tolerances of remanufacturing parts from the assembly angle in the actual production process. Remanufacturing parts' tolerance redistribution should comprehensively consider the damage degree of waste parts, the capability of the remanufacturing process, the precision of the remanufactured parts and so on to balance the cost and quality of the remanufactured products.

Learning from the above studies in the Introduction, we introduce the method overview in Section 1 . This paper uses an entropy model for measuring the uncertainty of assembly dimensional in Section 2. Then, in Section 3, a tolerance grading allocation method is proposed. Section 4 constructs the mathematical model of tolerance redistribution, including the processing precision tolerance redistribution restriction index, and the remanufactured parts' processing cost. Finally, Section 5 uses the remanufacturing assembly dimension chain of the automobile gear box as an example to demonstrate the feasibility and effectiveness.

\section{General Overview}

By the uncertainty principle $[20,21]$, it can be recognized that uncertainty is absolute, and determination is relative. Therefore, the uncertainty that exists in the remanufacturing also exists in the manufacturing, but the measured value of uncertainty is very different. The uncertainty of remanufacturing is higher than the remanufacturing. It is the key for optimizing decisions in the remanufacturing production process. Therefore, it requires a unified unit that is able to accurately measure these uncertainties, to achieve the quantification and contrast. In view of this, this paper is based on information entropy to quantify a description of the uncertainty of remanufactured parts, and the entropy is substituted by the assembly dimension chain to measure the uncertainty 
of the remanufacturing assembly. Related research literature $[5-8,10,11]$ shows that the value of the uncertainty of remanufacturing assembly is a quantitative description of the reassembly precision in the remanufacturing process, which largely reflects the quality of the remanufactured products.

In the remanufacturing assembly process, the reused parts, remanufactured parts and original parts in terms of quality and cost are different. It intensifies the instability of the assembly quality, and influences the precision of the remanufacturing assembly. On the other hand, by the promotion of environmental and economic benefits, remanufacturing engineering will save production costs and improve resource utilization as well as low carbon environmental protection as the main goal of production management. Therefore, how to maximize the use of different qualities of remanufacturing parts, and to ensure the accuracy of remanufacturing products, has become a challenge in manufacture assembly quality control. In order to achieve this goal, this paper proposes a tolerance redistribution optimization model of the reassembly dimensional chain. Its flow chart is shown in Figure 1.

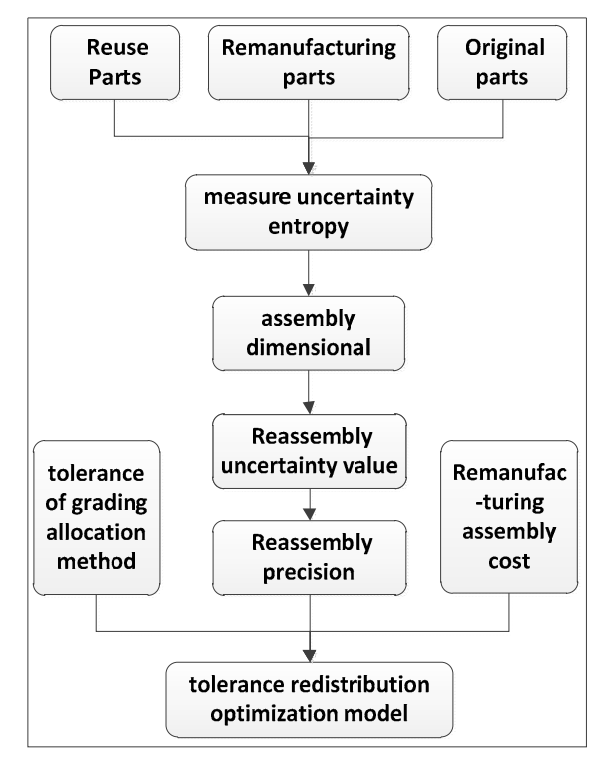

Figure 1. The flow chart of the tolerance redistribution optimization model.

\section{An Entropy Model for Measuring Uncertainty of Assembly Dimensional}

The parts' uncertainty is about the various parameters' uncertainty (randomness and fuzziness). The randomness has the same function in reflecting the uncertainty degree as the fuzziness, so we can use a composite entropy model to measure the uncertainty of the assembly dimensional.

The model assumes that the assembly dimensional of parts $X$ have $m$ attribute points, and there are $n$ states of each attribute point; $x_{i}$ is the $i$-th attribute point of part $X ; X_{i j}$ is the $j$-th state of the $i$-th attribute point; $p_{i j}$ is the a priori probability of $X_{i j}$ occurrence. The entropy of $X_{i j}$ can be expressed as follows:

$$
H\left(X_{i j}\right)=-\lambda P_{i j} \ln \left(P_{i j}\right)
$$

where $\lambda$ is a constant dependent on the chosen logarithm function, and $\lambda=1$.

So we can deduce the static entropy model for part $X$ :

$$
\begin{gathered}
H(x)=\sum_{i=1}^{m} \sum_{j=1}^{n} H\left(X_{i j}\right) \\
=-\sum_{i=1}^{m} \sum_{j=1}^{n} P_{i j} \ln \left(P_{i j}\right)
\end{gathered}
$$

where $P_{i j} \geq 0, \sum_{j=1}^{n} P_{i j}=1$. 
We do a comparison between the standards of the manufactured parts and the remanufactured parts. Assume that $\alpha_{i}{ }^{2}$ is the variance of the $i$-th attribute of manufactured part $X$ and $\beta_{i}{ }^{2}$ is the variance of the $i$-th attribute of remanufactured part $X$. Obviously, $0 \leq \alpha_{i} \leq \beta_{i}$.

According to the engineering practice experience, most of the performances (assembly size, stress, strength, fatigue, life, etc.) of the mechanical parts are in accordance with the normal distribution, the partial state distribution, the T distribution, and so on, but there are differences in the degree of dispersion, which shows that the uncertainty is different. With the entropy method, we define the assembly dimensional entropy as follows.

Thus, the entropy of manufactured parts is:

$$
H_{m}(x)=\sum_{i=1}^{m} \ln \sqrt{2 \pi} \alpha_{i}+C_{m}
$$

The entropy of remanufactured parts is:

$$
H_{r}(x)=\sum_{i=1}^{m} \ln \sqrt{2 \pi} \beta_{i}+C_{r}
$$

where $C_{m}$ and $C_{r}$ are constants.

In order to analyze the entropy of manufactured parts' assembly dimensional (MPAD) and remanufactured parts' assembly dimensional (RPAD), this paper assumes that the manufacturing assembly process and the remanufacturing assembly process were exactly same.

Due to $0 \leq \alpha_{i} \leq \beta_{i}$, we can infer the following results.

$$
\begin{gathered}
\ln \sqrt{2 \pi} \alpha_{i} \leq \ln \sqrt{2 \pi} \beta_{i} \\
\Rightarrow 0 \leq \sum_{i=1}^{m} \ln \sqrt{2 \pi} \alpha_{i} \leq \sum_{i=1}^{m} \ln \sqrt{2 \pi} \beta_{i} \\
\Rightarrow 0 \leq H_{m}(x) \leq H_{r}(x)
\end{gathered}
$$

Equation (7) shows that in accordance with the quality system and management methods of the manufactured assembly process, the entropy of remanufactured parts' assembly dimensions is higher, which leads to an increase in the entropy of remanufacturing the final product. Obviously, the result indicates that the assembly precision of the remanufactured product is not as good as that of the manufactured product.

\section{Tolerance Grading Allocation Method}

In order to ensure the quality of remanufacturing assembly, the standardization of the tolerance grading allocation method is mainly as follows:

(1) The performance of the parts in the tolerance grading allocation can meet the requirements of safety and reliability of the service of remanufactured products.

(2) The entropy of reassembly is less than that of assembly.

(3) The reassembly accuracy of the remanufacturing dimension chain is not less than that of the manufacturing dimension chain.

(4) The key and important parts remanufacturing is generally divided into two or three grades; noncritical parts remanufacturing is divided into three or four grades.

As a target to guarantee that remanufactured products are equal to or better than the manufactured products, we must deal with the entropy of remanufactured parts assembly dimensional, namely reducing assembly errors, and inputting the negative entropy. A tolerance grading allocation method is proposed as follows: 
Set up a chain of assembly dimensional that have $T$ assembly parts. $x(t)_{i}$ is the $i$-th attribute of $t$-th parts in the dimension chain.

The $t$-th manufactured parts assembly dimensional: $\alpha(t)_{i}{ }^{2}$ is the variance, and $\left[-\psi(t)_{i}, \psi(t)_{i}\right]$ is the tolerance range.

The $t$-th remanufactured parts assembly dimensional: $\beta(t)_{i}{ }^{2}$ is the variance, and $\left[-\xi(t)_{i}, \xi(t)_{i}\right]$ is the tolerance range.

Then $\psi(t)_{i}$ is the upper limit of the mechanical dimension tolerance of the $i$-th attribute of the $t$-th manufactured parts in the dimension chain; $-\psi_{i}$ is the lower limit of the mechanical dimension tolerance of the $i$-th attribute of $t$-th manufactured parts in the dimension chain; $\xi(t)_{i}$ is the upper limit of the mechanical dimension tolerance of the $i$-th attribute of $t$-th remanufactured parts in the dimension chain; $-\xi(t)_{i}$ is the lower limit of the mechanical dimension tolerance of the $i$-th attribute of $t$-th remanufactured parts in the dimension chain, where $\psi(t)_{i}>0, \xi(t)_{i}>0, i=1,2, \cdots, T$.

Suppose remanufacturing parts $x(t)$ are divided into $g(t)$ grades, the variance of the $i$-th attribute is $\beta(t)_{i}{ }^{2} / g(t)$, and the tolerance is also divided into $g(t)$ grades, respectively:

$$
\left\{\left[-\xi(t)_{i},-\xi(t)_{i}+2 \xi(t)_{i} / g(t)\right], \ldots,\left[\xi(t)_{i}-2 \xi(t)_{i} / g(t), \xi(t)_{i}\right]\right\}
$$

So, the entropy of the tolerance grading allocation for the remanufacturing assembly is:

$$
S_{r}=\sum_{t=1}^{T} \sum_{i=1}^{I} \ln e \sqrt{2 \pi / g(t)} \beta(t)_{i}
$$

The entropy of the tolerance grading allocation for the manufacturing assembly is:

$$
S_{m}=\sum_{t=1}^{T} \sum_{i=1}^{I} \ln e \sqrt{2 \pi} \alpha(t)_{i}
$$

In order to guarantee that the remanufactured assembly precision is not lower than the manufactured assembly precision, we just ensure that:

$$
S_{r} \leq S_{m}
$$

Namely,

$$
\sum_{t=1}^{T} \sum_{i=1}^{I} \ln e \sqrt{2 \pi / g(t)} \beta(t)_{i} \leq \sum_{t=1}^{T} \sum_{i=1}^{I} \ln e \sqrt{2 \pi} \alpha(t)_{i}
$$

It means that the $t$-th manufactured parts' grade number $g(t)$ should be larger than $\beta(t)_{i} / \alpha(t)_{i}$.

Through the above mathematical formula, and according to the actual production experience, the tolerances' and variances' liberalization coefficients of each classification for remanufactured parts are as shown in Table 1.

Table 1. Tolerances' and variances' liberalization coefficient of each classification.

\begin{tabular}{ccc}
\hline Class & Coefficient of Variance & Coefficient of Tolerance \\
\hline 2 & 1.75 & 1.25 \\
3 & 2.00 & 1.35 \\
4 & 2.25 & 1.45 \\
\hline
\end{tabular}

\section{The Tolerance Redistribution Optimization Model of the Reassembly Dimensional Chain}

In order to enhance assembly precision, to make sure that the product quality is not lower than that of the original product, this paper proposes the tolerance redistribution optimization model of the reassembly dimensional chain based on the tolerance of grading allocation method. On the 
basis of the quantitative measurement of uncertainty of the assembly dimension, it calculates the uncertainty of the remanufacturing assembly by the dimension chain. Furthermore, it quantifies the accuracy of the assembly. In the case that the uncertainty of the remanufacturing assembly is not lower than that of the original product assembly, the optimal assembly cost is provided, which provides a method and technical support for the production process management of the remanufacturing production enterprise.

\subsection{Constraint of Assembly Dimension Entropy}

The uncertainty of the assembly dimension is essentially a quantitative measure of the assembly precision. The constraint of the assembly dimension entropy means to ensure that the entropy of the reassembly dimension is not less than that of the assembly. In other words, it is better to make sure that the reassembly precision is better than that of the assembly.

First, we define the $j$-th grade for the $i$-th attribute of $t$-th parts in the dimension chain as $x(t)_{i j}$.

Namely,

$$
\beta(t)_{i j}=\beta\left[x(t)_{i j}\right]=\beta(t)_{i} / g(t)
$$

where $\beta(t)_{i j}{ }^{2}$ is the variance of $x(t)_{i j}$.

Similarly,

$$
\alpha(t)_{i 1}=\alpha\left[x(t)_{i 1}\right]
$$

There is only one grade of the original manufacturing parts, so $j=1$.

We can get:

$$
\sum_{t=1}^{T} \sum_{i=1}^{I} \ln e \sqrt{2 \pi} \beta(t)_{i j} \leq \sum_{t=1}^{T} \sum_{i=1}^{I} \ln e \sqrt{2 \pi} \alpha(t)_{i 1}
$$

Then, the constraint of the assembly dimension entropy is

$$
\sum_{t=1}^{T} \sum_{i=1}^{I} \ln e \sqrt{2 \pi} \beta\left[x(t)_{i j}\right] \leq \sum_{t=1}^{T} \sum_{i=1}^{I} \ln e \sqrt{2 \pi} \alpha\left[x(t)_{i 1}\right]
$$

\subsection{Constraint of Assembly Cost}

In this paper, we define the processing cost as the comprehensive cost including the processing time, equipment, difficulty coefficient, and so on. Because there are differences in the assembly operations of different grades of parts, it will inevitably lead to a difference in assembly cost.

So, the cost of the assembly dimension $x(t)_{i j}$ is as follows:

$$
C(t)_{i j}=c(t)_{i j} x(t)_{i j}
$$

where $c(t)_{i j}$ is the ratio of the assembly cost for $x(t)_{i j}$.

Then, the total processing cost of the assembly dimension chain is:

$$
C=\sum_{t=1}^{T} \sum_{i=1}^{I} \sum_{j=1}^{J} C(t)_{i j}=\sum_{t=1}^{T} \sum_{i=1}^{I} \sum_{j=1}^{J}\left[c(t)_{i j} x(t)_{i j}\right]
$$

\subsection{The Tolerance Redistribution Optimization Model}

The tolerance redistribution optimization model of the reassembly dimensional chain is implemented at the same time to ensure that the assembly precision of the remanufactured product is not less than that of the original product, and the optimization of the assembly cost can be realized. 
Considering the uncertainty of the assembly process and the assembly precision of the complex mechanical product, we set up the tolerance redistribution optimization model of the reassembly dimensional chain as follows:

$$
\begin{aligned}
& \min C=\sum_{t=1}^{T} \sum_{i=1}^{I} \sum_{j=1}^{J}\left[c(t)_{i j} x(t)_{i j}\right] \\
& \text { s.t }\left\{\begin{array}{l}
\sum_{t=1}^{T} \sum_{i=1}^{I} \ln e \sqrt{2 \pi} \beta\left[x(t)_{i j}\right] \leq \sum_{t=1}^{T} \sum_{i=1}^{I} \ln e \sqrt{2 \pi} \alpha\left[x(t)_{i 1}\right] \\
\sum_{j=1}^{I} x(t)_{i j}=1 \\
x(t)_{i j}=0 \text { or } 1
\end{array}\right.
\end{aligned}
$$

The model is a minimum of linear integer programming problem, which belongs to the $0 / 1$ Knapsack Problem, there are many methods to solve it. Therefore, this article will not go into the details.

\section{Case Study}

A certain company is mainly engaged in the auto parts remanufacturing processing business. The main products are remanufactured gearboxes, remanufactured engines, and so on. The enterprise production statistics show that in the remanufactured gearbox, more than $70 \%$ of the parts are remanufactured parts, but the one-time assembly pass rate is only about $86 \%$. The repair rate is high, the cost of the customer service is large, and customer complaints are frequent. In the years 2013 and 2014, sales service costs were as high as 4.83 million RMB, accounting for $8.4 \%$ of the sales amount. It is a difficult problem for the enterprise to use the limited precision of the remanufactured parts to assemble the high-quality remanufactured gearbox in the production process management. In order to solve this problem, the tolerance redistribution optimization model of the reassembly dimensional chain is put forward.

\subsection{Applications on a Remanufactured Gearbox}

In order to describe the operation and solving process of the model, we take a dimensional chain of the remanufactured gearbox (RHGB-6028 as an example, which is in Figure 2).

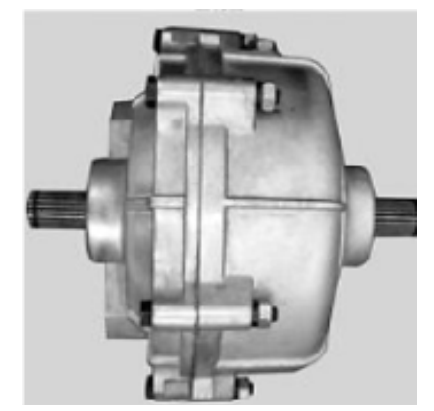

(a)

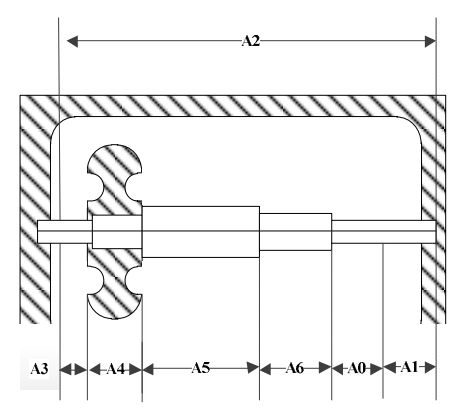

(b)

Figure 2. The remanufactured gearbox.

Among them, Figure 2a is the RHGB-6028 remanufactured gear box, and Figure $2 b$ is the $2 \mathrm{D}$ structure diagram. With the help of Figure 1, we can get a dimension chain as shown in Figure 3. 


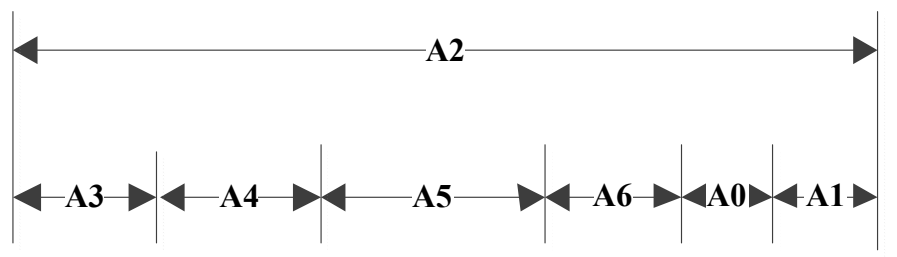

Figure 3. The remanufactured gearbox dimension chain graph.

This chain is composed of seven dimensional (A0, A1, A2, A3, A4, A5 and A6), and their relationship is clear. Dimensional tolerances' classification and their processing cost of the manufactured gearbox parts and the remanufactured gearbox parts are listed in the Table 2 .

Table 2. Dimensional tolerances' classification and their processing cost.

\begin{tabular}{|c|c|c|c|c|c|c|c|c|}
\hline \multirow{3}{*}{ Parts } & \multirow{3}{*}{$\begin{array}{c}\text { Basic } \\
\text { Dimensional }\end{array}$} & \multirow{3}{*}{$\begin{array}{c}\text { Manufacturing } \\
\text { Tolerance }\end{array}$} & \multicolumn{6}{|c|}{ Remanufacturing } \\
\hline & & & \multicolumn{2}{|c|}{ First Grade } & \multicolumn{2}{|c|}{ Second Grade } & \multicolumn{2}{|c|}{ Three Grade } \\
\hline & & & Tolerance & Cost & Tolerance & Cost & Tolerance & Cost \\
\hline A0 & 5 & 0.02 & 0.02 & 1.5 & 0.03 & 1.2 & 0.04 & 1 \\
\hline A1 & 20 & 0.05 & 0.05 & 2 & 0.06 & 1.8 & 0.07 & 1.5 \\
\hline A2 & 155 & 0.1 & 0.1 & 8 & 0.12 & 7.5 & 0.14 & 6 \\
\hline A3 & 15 & 0.05 & 0.05 & 1 & 0.06 & 0.8 & 0.07 & 0.6 \\
\hline A4 & 40 & 0.05 & 0.05 & 3 & 0.06 & 2.5 & 0.07 & 2 \\
\hline A5 & 50 & 0.05 & 0.05 & 5 & 0.06 & 4.5 & 0.07 & 4 \\
\hline A6 & 25 & 0.03 & 0.03 & 2 & 0.04 & 1.8 & 0.05 & 1.5 \\
\hline
\end{tabular}

In the actual production process, the dimensional tolerances of remanufactured parts are divided into several grades depending on the precision of each classification. Here, we assume that they are divided into three grades.

In order to facilitate the calculation, we make the tolerance in $\mathrm{mm}$.

The entropy value of manufactured products: $S_{m}=3.8313 \mathrm{bit}$.

The entropy value of remanufactured products: $S_{r}=3.6750 \mathrm{bit}$.

So, $S_{r}<S_{m}$.

If the remanufactured parts are processed according to the standards of the manufactured parts, their total processing cost is $c_{m}=22.5$.

The software of LINGO11.0 is applied to construct and solve the model on the dual-core computer (processor: $3.0 \mathrm{GHz}$, memory: $2 \mathrm{G}$ ). The solver status window is as shown in Figure 4.

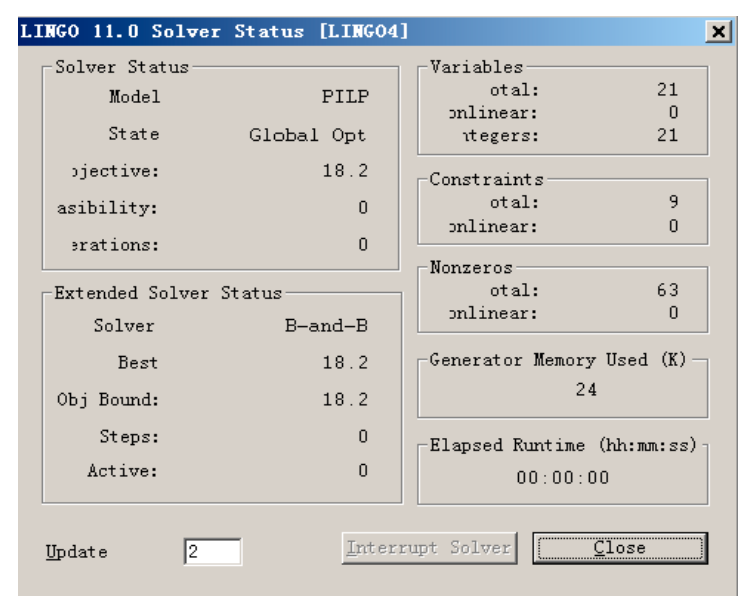

Figure 4. Solver status window. 
As we can see from Figure 4, the model belongs to the pure integer linear programming problem (PILP), and the global optimum solution is obtained and the optimal objective value is 18.2. The dimensional tolerance optimal redistribution scheme of the remanufactured parts based on the optimal objective value is calculated (operation results are shown in Table 3). The cost of the remanufactured assembly processing saves $19.11 \%$ after adopting the tolerance redistribution optimization model.

Table 3. The tolerance redistribution optimization scheme.

\begin{tabular}{cccc}
\hline Parts & First Grade & Second Grade & Three Grade \\
\hline A0 & 0 & 1 & 0 \\
A1 & 1 & 0 & 0 \\
A2 & 0 & 0 & 1 \\
A3 & 1 & 0 & 0 \\
A4 & 0 & 0 & 1 \\
A5 & 0 & 0 & 1 \\
A6 & 1 & 0 & 0 \\
\hline
\end{tabular}

\subsection{Comparison}

The tolerance redistribution optimization model for the remanufactured gearbox was implemented in 2015. It improved the quality of the assembly of the gearbox effectively. Statistics in 2015 showed that compared with 2014, the pass rate of the remanufactured gearbox increased by an average of $2.16 \%$ (Figure 5).

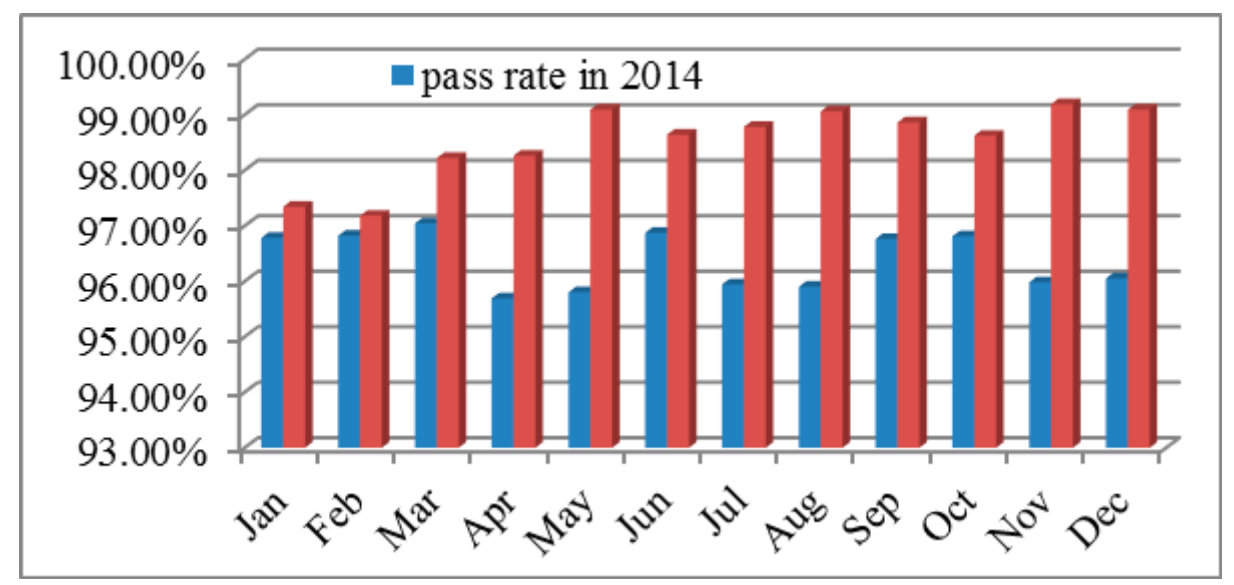

Figure 5. Pass rate in 2014 and 2015.

In this model, the reassembly cost is minimized, and the coupling of the dimension chain is constrained by the entropy. This model has the following advantages in enterprise applications:

(1) In the case of ensuring that the reassembly accuracy is not lower than that of the original product, it can increase the utilization rate of the remanufactured parts, and decrease the production cost.

(2) It can reduce the influence of the uncertainty of the remanufacturing assembly process, improve the reassembly precision, and improve the quality of remanufactured products.

(3) It enhances the management level of the remanufacturing production process, improves the assembly qualified rate, reduces the cost after sales services and increases customer satisfaction.

(4) It can also reduce the occurrence of accidents and improve the level of production safety management. 


\section{Conclusions}

How to expand the utilization rate of remanufactured parts and improve the reassembly precision is a scientific challenge. In order to solve this problem, this paper has done the following innovative work:

(1) We established the entropy model to measure the uncertainty of assembly dimensional, and explored the assembly process, which proves that the remanufactured assembly uncertainty is higher than the manufactured assembly uncertainty, and it can quantify the accuracy of the assembly.

(2) In order to enhance the assembly precision, we make sure that the product quality is not lower than that of the original product. The tolerance redistribution optimization model of the reassembly dimensional chain based on the tolerance of grading allocation method is proposed.

(3) We apply this method in the remanufactured gearbox, which is showing that there is a great benefit in expanding the utilization rate of remanufactured parts and improving the reassembly precision.

The uncertainty optimization theory of remanufacturing assembly is a multidisciplinary, interdisciplinary and comprehensive theory. In this paper, we define, measure and optimize the uncertainty of remanufacturing assembly from the point of view of entropy. In order to further study the uncertainty of remanufacturing assembly, we will adopt the theory of fuzzy mathematics, the rough set theory and the cloud model to enrich and perfect the theory of uncertainty in remanufacturing assembly.

Acknowledgments: This research is supported by the National Basic Research Program (973 Program): fundamental science research of mechanical equipment remanufacturing (project number: 2011CB013406) and the Anhui University Natural Science Research Project (KJ2016A776).

Conflicts of Interest: The authors declare no conflict of interest.

\section{References}

1. $\mathrm{Xu}, \mathrm{B}$. Remanufacturing and Recycling Economy; Science Press: Beijing, China, 2007. (In Chinese)

2. Liu, M.; Liu, C.; Ge, M.; Zhang, Y.; Liu, Z. The online quality control method for reassembly based on state space model. J. Clean. Prod. 2016, 137, 644-651. [CrossRef]

3. Fang, J.X.; Dong, S.Y.; Wang, Y.J.; Xu, B.S.; Zhang, Z.H. The effects of solid-state phase transformation upon stress evolution in laser metal powder deposition. Mater. Des. 2015, 87, 807-814. [CrossRef]

4. Wei, S.; Liu, Y.; Tian, H.; Tong, L.; Liu, Y.; Xu, B. Microwave absorption property of plasma spray W-type hexagonal ferrite coating. J. Magn. Magn. Mater. 2015, 377, 419-423. [CrossRef]

5. Liu, M.; Liu, C.; Zhu, Q. Optional classification for reassembly methods with different precision remanufactured parts. Assem. Autom. 2014, 34, 315-322. [CrossRef]

6. Liu, C.; Liu, M.; Xing, L. A research on the uncertainty measure and its application to the journal surface roughness of remanufactured engine crankshaft. Automot. Eng. 2015, 37, 341-345.

7. Liu, M.; Liu, C.; Xing, L.; Mei, F.; Zhang, X. Study on a tolerance grading allocation method under uncertainty and quality oriented for remanufactured parts. Int. J. Adv. Manuf. Technol. 2013, 2013. [CrossRef]

8. Ge, M.; Liu, C.; Liu, M. The online quality control methods for the assembling of remanufactured engines' cylinder block and cover under uncertainty. Int. J. Adv. Manuf. Technol. 2014, 74, 225-233. [CrossRef]

9. Jin, X.; Hu, S.J.; Ni, J.; Xiao, G. Assembly strategies for remanufacturing systems with variable quality returns. IEEE Trans. Autom. Sci. Eng. 2013, 10, 76-85. [CrossRef]

10. Liu, M.; Liu, C.; Xing, L.; Liu, Z.; Li, X.; Lin, L. Assembly process control method for remanufactured parts with variable quality grades. Int. J. Adv. Manuf. Technol. 2016, 85, 1471-1481. [CrossRef]

11. Liu, M.; Liu, C.; Xing, L.; Zhang, X.; Wang, Q. Quality Oriented Assembly Grouping Optimal Allocation Method for Remanufactured Complex Mechanical Products. J. Mech. Eng. 2014, 50, 150-155. [CrossRef]

12. Shannon, C.E. A Mathematical Theory of Communication. Bell Syst. Tech. J. 1948, 27, 379-423, $623-656$. [CrossRef] 
13. Chryssolouris, G.; Vassiliou, E.; Mavrikios, D. Application of Information Theory to the Quantification of Concurrent Engineering Processes. In Leading the Web in Concurrent Engineering: Next Generation Concurrent Engineering; IOS Press: Amsterdam, The Netherlands, 2006.

14. Frizelle, G.; Suhov, Y.M. An entropic measurement of queueing behaviour in a class of manufacturing operations. Proc. R. Soc. A 2001, 457, 1579-1601. [CrossRef]

15. Efstathiou, J.; Calinescu, A.; Blackburn, G. A web-based expert system to assess the complexity of manufacturing organizations. Robot. Comput. Integr. Manuf. 2002, 18, 305-311. [CrossRef]

16. Kuzgunkaya, O.; ElMaraghy, H.A. Assessing the structural complexity of manufacturing systems conFigureurations. Int. J. Flex. Manuf. Syst. 2006, 18, 145-171. [CrossRef]

17. Cho, S.; Alamoudi, R.; Asfour, S. Interaction-based complexity measure of manufacturing systems using information entropy. Int. J. Comput. Integr. Manuf. 2009, 22, 909-922. [CrossRef]

18. Zhang, Z. Complexity of cellular manufacturing systems based on entropy models. J. Mech. Sci. Technol. 2010, 24, 2275-2280. [CrossRef]

19. Duan, J.; Li, A.; Xie, N.; Xu, L. Structural Complexity Modeling and Evaluation of Manufacturing Systems Based on State Entropy. J. Mech. Eng. 2012, 48, 92-100. [CrossRef]

20. Robertson, H.P. The Uncertainty Principle. Nature 2004, 431, 487-487. [CrossRef]

21. Zhang, C.; Liu, C.; Liu, L. Diagnosis and application of carbon footprint for machining workshop on energy saving and emission reduction. Comput. Model. New Technol. 2014, 18, 265-270.

(C) 2016 by the author; licensee MDPI, Basel, Switzerland. This article is an open access article distributed under the terms and conditions of the Creative Commons Attribution (CC-BY) license (http://creativecommons.org/licenses/by/4.0/). 